(C) 1996 IEEE. Personal use of this material is permitted. However, permission to reprint/republish this material

for advertising or promotional purposes or for creating new collective works for resale or redistribution to servers

or lists, or to reuse any copyrighted component of this work in other works must be obtained from the IEEE.

\title{
THE AMPS RING: ACTUAL PERFORMANCE AND FUTURE PLANS
}

G. Luijckx, R. Bakker, H. Boer Rookhuizen, C. de Jager, F. Kroes, L. Kuijer, J. van der Laan, R. Maas, J. Noomen, Y. Wu, NIKHEF, P.B. 41882, 1009 DB Amsterdam, the Netherlands

The Amsterdam Pulse Stretcher AmPS is a $300-900 \mathrm{MeV}$ electron storage ring with a circumference of $212 \mathrm{~m}$. The ring operates either in stretcher mode to provide external continuous beams of tens of $\mu \mathrm{A}$ or in storage mode with currents up to $200 \mathrm{~mA}$ for internal target experiments. Machine commissioning and simultaneous operation started for nuclear physics started mid 1992. The actual performance is presented. During 1995 a polarized electron source will be added to the linac injector. A "Siberian Snake" acting as a spin flipper will be implemented in the ring; these systems have been made by the Institute for Semiconductor Physics at the Budker Institute of Nuclear Physics both at Novosibirsk as part of a scientific collaboration agreement. Results from a feasibility study on the use of the ring as a free electron laser in the VUV region are also summarized.

\section{INTRODUCTION}

NIKHEF is a nuclear physics and high-energy physics research institute. Since the early eighties the nuclear physics branch is specialized in electron scattering experiments using several detectors measuring in coincidence. Electron beams with a high duty factor (d.f.) are required to obtain a good real to accidental coincidence ratio. Initially electrons were provided by the $500 \mathrm{MeV} 1 \%$ d.f. linac MEA [1]. Meanwhile the pulse stretcher ring AmPS was designed [2] with the aim to improve the duty factor with almost 2 orders of magnitude. Installation of the AmPS facility started early 1991 and the first extracted beam on target was available by mid 1992. The optical design and the first commissioning results were reported earlier [3-5]. The facility is also used in storage mode for experiments with internal targets. Experiments with stored polarized electrons are scheduled from $1996 \mathrm{on}$. Already before the completion of AmPS the funding agency F.O.M. announced to dramatically reduce the funding of nuclear physics research in the Netherlands from mid 1998 on. As a result the AmPS facility will only be available for nuclear physics until that date. There is obviously a strong pressure on the accelerator group to deliver as much beam as possible for nuclear physics often at the detriment of proper commissioning and accelerator development. Meanwhile plans are developed to convert the AmPS ring to a free electron laser operating in the V.U.V. wavelength after 1998 .

\section{A. General}

\section{PERFORMANCE}

AmPS is now in the production phase were it routinely delivers beams for nuclear physics.. The overall reliability has been improving gradually since the first external beam with a high duty factor was generated in 1992. In 1994 beam was available for experiments during 2200 hours: $575 \mathrm{~h}$ in storage mode, $1250 \mathrm{~h}$ in stretcher mode and 375 hours for commissioning. The unscheduled downtime was $10 \%$. Once tuned the ring operation normally remains extremely stable during many days but tuning of the beam, especially in stretcher mode, is still an expert process.

Table 1 shows that the obtained beam parameters so far come close to their target values.

\begin{tabular}{|c|c|c|c|}
\hline $\begin{array}{c}\text { Operation } \\
\text { mode }\end{array}$ & $\begin{array}{c}\text { Energy } \\
(\mathrm{MeV})\end{array}$ & $\begin{array}{c}\text { Current } \\
(\mathrm{mA})\end{array}$ & $\begin{array}{c}\text { Duty factor } \\
(\%)\end{array}$ \\
\hline Storage & 635 & 150 & 100 \\
(internal targets) & $\mathbf{8 5 0}$ & $\mathbf{2 0 0}$ & $\mathbf{1 0 0}$ \\
Stretcher & 600 & $10 \mu \mathrm{A}$ & 94 \\
(external targets) & $\mathbf{7 0 0}$ & $\mathbf{2 0 \mu A}$ & $>\mathbf{8 0}$ \\
\hline
\end{tabular}

Table 1. AmPS beam parameters available on the experimental targets (as achieved until May 1995 ).

The goals are displayed in bold.

\section{B. Linac}

The electron linac MEA operated as a $1 \%$ duty factor (d.f.) $500 \mathrm{MeV}$ machine from 1980 to 1991 . Its modulatorklystron units were then modified to provide energies up to $900 \mathrm{MeV}$ at a d.f. of $0.1 \%$ [6]. The maximum energy the linac can provide at $\mathrm{i}=0$ is now $770 \mathrm{MeV}$.

The 520 pulse forming network (pfn's) units of MEA show some degradation after 15 year of operation. Presently all pfn's are successively refurbished.

Accelerating 0.7 to $2.1 \mu$ s beam pulses at currents up to $50 \mathrm{~mA}$ requires compensation of the transient beam loading because of the $1.2 \mu \mathrm{s}$ fill time of the accelerator sections and the beam loading of $2.6 \mathrm{MeV} / \mathrm{mA}$. The compensation technique is based on staggered triggering of individual modulator RF pulses. At MEA the RF triggering of each station can be altered in steps of $10 \mathrm{~ns}$. This compensation method is in use for several years and can "compress" 130 $\mathrm{MeV}$ energy spread to approximately $5 \mathrm{MeV}$ by shifted RF triggering on 4 of the 12 klystron modulators. To work satisfactorily the timing generators have to be very stable and the beam transport in the linac has to be well centered to avoid energy dependent steering by the quadrupoles.

Originally MEA was designed and constructed as a $10 \%$ d.f. accelerator. This high d.f. required intensive cooling amongst others of the pfn's. FREON 113, a Carbon Fluor Chlorine (CFC) was chosen as a coolant and was sprayed on the pfn's. Although the present d.f. of MEA is limited to .1\% cooling of the pfn's is still required. Because of environmental requirements the use of $\mathrm{CFC}$ is no longer allowed in the Netherlands. Perchloroethane (p.e.r.) showed to be an acceptable substituent and the cooling systems have been adapted accordingly.

\section{Ring}

1. Operational aspects

Stretcher mode: one turn injection provides a very high 
quality beam with a macroscopic d.f. well over $90 \%$. Fig.1 shows a typical plot of the circulating and extracted current. When the nuclear physics detectors operate at their maximum time resolution ( nanoseconds) they "see" a time structure in the beam that corresponds to the revolution frequency ; this effect reduces the d.f. to $70 \%$. Operation up to 3 turn injection at a horizontal tune $\left(v_{\mathrm{X}}\right)$ of 8.3 is achieved on a regular basis but requires sensitive tuning of machine to avoid beamloss. Also the d.f. is still somewhat lower, $50 \%$. Stretcher operation at a $v_{\mathrm{X}} \sim 8.25$ allowing 4 turn injection has been demonstrated but requires further investigation to ensure sufficient clearance of the beam from the injection septum. The extraction is controlled by 4 extraction sextupoles in the straight sections and by the $2856 \mathrm{MHz} \mathrm{RF}$ system. Until now only the amplitude of the RF is modulated but in the future phase modulation will be investigated too.

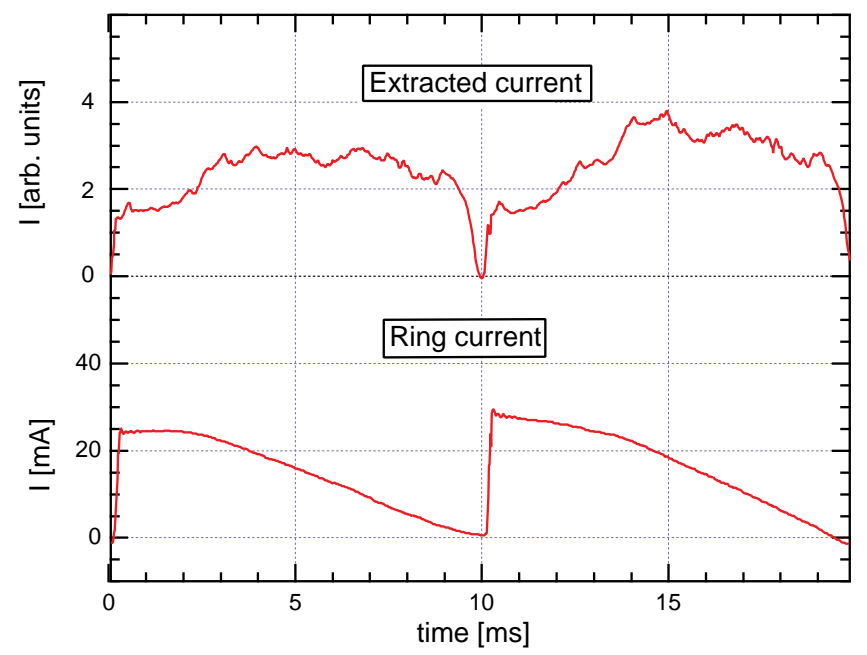

Fig.1. Typical current patterns in stretcher mode.

Storage mode: currents up to $150 \mathrm{~mA}$ have been stored an the maximum energy obtained was $630 \mathrm{MeV}$. Lifetime (1/e) is in the order of 30 minutes and is limited by the relatively high vacuum pressure of $1 * 10^{-7}$ mbar @ $100 \mathrm{~mA}$ beam. This lifetime is sufficient for internal target physics purposes. Up to $500 \mathrm{MeV} \mathrm{RF}$ acceleration is ensured by a $2856 \mathrm{MHz}$ system [7\&8]. This system normally operates at $30 \mathrm{~kW}$ at which level it provides an acceleration voltage of $130 \mathrm{kV}$. A $476 \mathrm{MHz}$ system is available since early 1995 to allow operation beyond $500 \mathrm{MeV}$ [9]. It consists of a modified single cell Doris cavity and a CW $476 \mathrm{MHz} 30 \mathrm{~kW}$ transmitter. The ring can be used as a synchrotron to enable to operate at energies above the injection energy. This way an energy of $630 \mathrm{MeV}$ was obtained with an injection energy of $330 \mathrm{MeV}$. The ramping speed is limited by the data transfer rate of the magnet power supplies. To eliminate this bottleneck part of the serial communication links will be converted to parallel communication in the future.

Circulating currents above $100 \mathrm{~mA}$ have been obtained by stacking the injection pulses. Apparently because of the poor vacuum the maximum current is limited to $\sim 150 \mathrm{~mA}$. At this current and pressure ions are clearly present and the clearing electrodes have to be powered $(4 \mathrm{kV})$. Also tune shifts as function of the stored current have been noticed. Partial filling of the ring will be tested to increase the maximum current.

\section{Machine parameters}

Both beta and dispersion functions as obtained from the machine tuning procedure have been measured recently [10]. It appeared that especially the dispersion function deviated strongly from theory: there was even some dispersion in the straights while they were designed to be dispersion free . After a 1 to $2 \%$ correction of the calculated quadrupole settings the machine functions are now close to their theoretical values. The circumference of the central orbit has been measured and appears to be $\sim 1 \mathrm{~cm}$ shorter than the required $211.618 \mathrm{~m}$ to fit 2016 buckets. In storage mode this effect is counteracted by a slightly higher oscillator frequency. In stretcher mode the RF is locked to the linac frequency so the closed orbit can't follow the central trajectory in the magnets . The linac frequency therefore will be adapted.

3. Hardware performance

Magnets [11], septa [12] and their power supplies show a very good long-term stability and operate very reliably. Also both the $2856 \mathrm{MHz}$ and the $476 \mathrm{MHz} \mathrm{RF}$ accelerator systems operate according to specification. From Fall 1995 the performance of the fast switching kicker power supplies [13] will be enhanced through new deflector insulators and improved pulse power electronics .

Diagnostics: an overview is available in ref [14]. A major drawback is the lack of a reliable closed orbit correction (c.o.c.) tool. The beam position information for the present tool comes from the $2856 \mathrm{MHz}$ stripline monitors [15]. Because the cut-off frequency of the beam pipes is $>2856$ $\mathrm{MHz}$ the slm's are sensitive to wake fields and require lengthy and tedious calibration of both sensitivity and offset at regular intervals. A new c.o.c. tool is now being developed based on the use of wobbling quadrupoles as beam position monitors [16]. Ion chamber based radiation loss detectors are very helpful in minimizing the beam loss around the ring. This is particularly important in stretcher mode when the average beam power can be as high as $15 \mathrm{~kW}$.

Vacuum: the present pump capacity is based on stretcher mode operation only and is marginal for storage mode at high energy and with high currents. So NEG strips from SAES will be implemented inside of the Varian Star cell pumps mid 1995 to improve the pumping by at least an order of magnitude for light molecules $\left(\mathrm{H}_{2}\right)$

\section{A. Polarized electrons}

\section{FUTURE}

A design for producing a stored beam of longitudinally polarized electrons has been made by NIKHEF in collaboration with the BINP and ISP institutes from Novosibirsk. The polarized electrons are produced by illuminating a strained GaAs photo cathode with circularly polarized light from a flash lamp pumped pulsed $5 \mathrm{~kW}$ Tisapphire laser. The polarization vector can be rotated to an arbitrary angle with a Z-shaped manipulator consisting of two 
electrostatic deflectors and eight solenoids. The polarization degree can be measured with a Mott polarimeter. A $100 \mathrm{keV}$ electron beam with a peak current of $40 \mathrm{~mA}$ and a pulse length of $2 \mu \mathrm{s}$ is extracted from the source at a maximum repetition rate of $2 \mathrm{~Hz}$. A two-cavity scheme, one for bunching the electron beam coming out from the Zmanipulator and another for acceleration to $400 \mathrm{keV}$ will be incorporated between the polarized electron source and MEA With this design both the polarized and the existing thermionic source can be used alternatively. The expected capture efficiency of $20 \%$ results in an $8 \mathrm{~mA}$ peak current in MEA. By three-turn injection $20 \mathrm{~mA}$ is then captured in the AmPS ring. Consecutive pulses accelerated in MEA are stacked into the ring until the desired intensity of over 100 $\mathrm{mA}$ is reached. A beam with energy up to $700 \mathrm{MeV}$ can be injected directly into the ring. The stored beam can also be ramped to a maximum energy of $900 \mathrm{MeV}$.

In order to maintain the polarization longitudinal at the interaction point, a Siberian Snake, consisting of two superconducting solenoids, two pairs oSf skew quadrupoles and one normal quadrupole will be installed in the East straight section of the AmPS ring. The degree of polarization of the stored beam will be measured by using a Compton back-scattering polarimeter, utilizing circularly polarized light at a wavelength of $528 \mathrm{~nm}$ produced by a $10 \mathrm{~W}$ Ar-ion laser in $\mathrm{CW}$ mode. Part of this work was funded by the Human Capital and Mobility program of the EEC under contracts numbered ERBCHBICT930606 and CHRX-CT93-0122.

\section{B. High luminosity.}

Luminosity for internal target experiments can be increased by reduction of both the target cell diameter and the beamsize at the IT. The present emittance is $96 \pi \mathrm{mm}$. mrad at $700 \mathrm{MeV}$. By lowering the dispersion function $\eta$ in the curves the emittance becomes $32 . \pi \mathrm{mm}$.mrad. Of course the Twiss parameters in the curves change and they have to be matched with the straight parameters. Fortunately this results in a decreased $\beta$ function value at the IT location. In total the beam diameter should be reduced by a factor of 2.7. By splitting the quadrupoles in 3 instead of 2 families this high luminosity scheme can be achieved. This requires only one additional power supply and the associated (re)cabling.

\section{Free electron laser}

Early 1995 a feasibility study [17] to incorporate a FEL in one of AmPS' straight sections was completed with help of Prof.. V. Litvinenko from the DFELL in Duke. It is shown that this FEL could operate in the Vacuum Ultra Violet (V.U.V.) at wavelengths below $100 \mathrm{~nm}$. To ensure sufficient gain for the lasing in this wavelength region the emittance of AmPS will have to be reduced and also the peak bunch current should be increased. As a first step a 'pilot experiment' has been proposed in which two $1.3 \mathrm{~m}$ undulators will be used (as an optical klystron) in conjunction with a reduced-emittance confi-guration of AmPS. It is expected that coherent radiation in the $250 \mathrm{~nm}$ range can be produced in this set-up. The two 24 pole electromagnetic undulators with a $\lambda_{\mathrm{W}}=11 \mathrm{~cm}$ are on loan from the Budker Institute of Nuclear Physics of Novosibirsk. By reducing the emittance of AmPS to the $10 \mathrm{~nm}$ rad @ E=900 MeV and extending the undulator length to the range of $8-15 \mathrm{~m}$, lasing should be feasible down to the $25 \mathrm{~nm}$ level. A first zero-order investigation how to modify the ring lattice to enable a low emittance has been already been made [18] but although apparently feasible further detailed analysis is clearly required.

\section{CONCLUSION}

The AmPs facility now operates almost completely according to the specifications required for nuclear physics experiments. Both for the near and the far future accelerator physics fun is ensured by challenging new projects.

\section{REFERENCES}

[1] C. de Vries et al., "The $500 \mathrm{MeV}$ Electron-Scattering Facility at NIKHEF-K" , Nucl. Instr. \& Meth., 223 ,pp. 1-25.

[2] G. Luijckx et al.," The Amsterdam Pulse Stretcher Project (AmPS)", Proc. 1989 Part. Accel. Conf., pp. 46

[3] R. Maas \& Y. Wu," Optics of the Amsterdam Pulse Stretcher", Proc. 1989 PAC., Chicago, pp. 1689

[4] G. Luijckx et al., "The Amsterdam Pulse Stretcher, first commissioning results", Proc. 1992 HEACC, pp. 464

[5] R. Maas et al., "Commissioning results of the AmPS Ring", Proc. 1993 PAC, Washington, pp. 1998

[6] F.B. Kroes, E. Heine, " Modification of MEA modulatorklystron units enabling short pulse injection into a pulsestretcher ring", Proc. 1989 PAC, pp. 205

[7] J. Haimson \& B. Mecklenburg, "Design and Operating Characteristics of a CW non-synchronous traveling wave structure for a $900 \mathrm{MeV}$ pulse stretcher ring", Proc. of the 1992 LINAC Conf., Ottawa

[8] F. Kroes et al., "A Fast Amplitude and Phase Modulated RF Source for AmPS", Proc. 1991 PAC, , pp. 684.

[9] F. Kroes et al., "A $476 \mathrm{MHz}$ RF system for the storage mode of the AmPS ring", this conference.

[10] Y.Y. Wu \& R. Maas, "Machine parameter measurement of the Amsterdam Pulse Stretcher AmPS", this conference.

[11] H. Boer Rookhuizen et al., "Magnetic Devices of the Amsterdam Pulse Stretcher Ring AmPS", Proc. 1991 PAC, San Francisco, pp. 2366.

[12] A. van der Linden et al., "High Power Density, Thin Magnetic DC. Septa for AmPS", Proc. EPAC 92, pp. 1478.

[13] E. Heine et al., "Pulsed Electrostatic Kickers with Low Beam Impedance for AmPS", Proc. EPAC 92, pp. 1475

[14] J.G. Noomen et al., "Beam diagnostics for the Amsterdam Pulse Stretcher AmPS", this conference

[15] J. Noomen et al., "An over-moded stripline beam position monitor", Proc. 1993 PAC., Washington, pp. 684.

[16] P. Röjsel, "A beam position measurement system using quadrupole magnets magnetic centra as the position reference", Nucl. Instr. \& Meth., 343 (1994) pp. 374.

[17] R.J. Bakker et al.," Expected Performance of FELINA, the Dutch VUV-FEL in Amsterdam ", Nucl. Instr. \& Meth.in Phys. Res., 358 (1995) pp. 358.

[18] R. Maas \& G. Luijckx, "AmPS, the Amsterdam Pulse Stretcher, as Photon Source", Proc. 1992 EPAC, pp. 480. 